
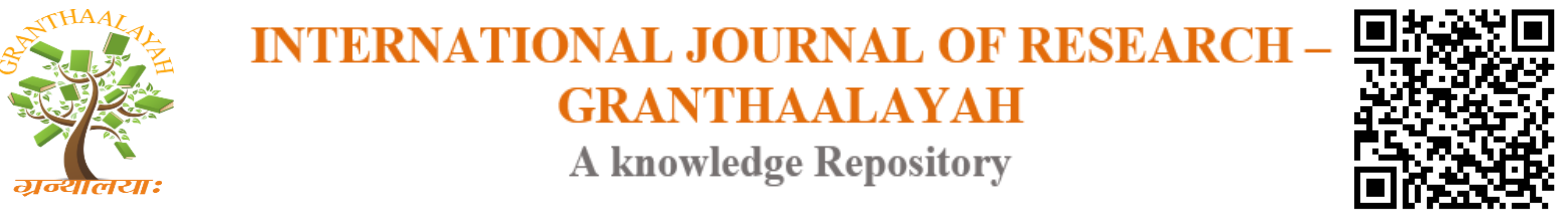

Science

\title{
ASSESSMENT OF MALARIA PREVENTION AND CONTROL METHODS IN SHENDI LOCALITY, SUDAN 2015
}

\author{
Dr. Omar Yousof Mohamed Ali ${ }^{* 1}$ \\ ${ }^{* 1}$ Assistant Professor, Faculty of Public Health, Shendi University, SUDAN
}

DOI: https://doi.org/10.29121/granthaalayah.v4.i6.2016.2648

\section{ABSTRACT}

This paper represented the founding of community based cross sectional study conducted to assess methods of prevention and control of malaria in Shendi city. Specifically it aims to evaluate the different methods of prevention service offered by local health authorities. Evaluation of community participation towards control of malaria and evaluation of personal protection against mosquito bite. Sample size of 192 was selected randomly out of 9000 households. An interview' questionnaires and observations were used to collect the data from households, then the data was analyzed using both Microsoft Excel and Statistic Package for Social Sciences program (SPSS).The main results showed that Most dominant level of education of shendi population was university and secondary school. The study concludes that most population used personal protection against mosquito bites.

Keywords:

Methods, prevention, control, Malaria, community.

Cite This Article: Dr. Omar Yousof Mohamed Ali, "ASSESSMENT OF MALARIA PREVENTION AND CONTROL METHODS IN SHENDI LOCALITY, SUDAN 2015" International Journal of Research - Granthaalayah, Vol. 4, No. 6 (2016): 150-156.

\section{INTRODUCTION}

Malaria is a preventable and curable disease and yet more than one million people die from it each year. It is a disease that significantly affects the poor who suffer economic, social and educational deprivation. Malaria is one of the most successfully parasites ever known to mankind After thousands of years it remains the world's most pervasive infection, affecting at least about 300-500milion clinical cases each year, with $90 \%$ of these occurring is sub-Saharan African and mostly caused P Falciparum.(National protocol for treatment of malaria,2010) .

(WHO, 2014) The World Health Organization (WHO) stated that, approximately $40 \%$ of the world's population in developing countries at risk of malaria Tropical, with the majority of the cases found in the developing world (WHO, 2007). Each year over 500 million people become ill with the disease and between 700,000 t0 2.7 million people, mostly children, die as a result in 
addition to the human health toll, the disease is also associated with significant economic losses, estimated at some US12 billion annually for sub-Saharan Africa alone (WHO, 2000; Gallup and Sachs, 2001 ; Amerasinghe, 2006; WHO,2007).

Malaria is a complex disease. Its severity is a function of the interaction between the parasite, the Anopheles mosquito vector, the human host and the environment. The risk of malaria infection is determined by the number of vectors, their survival rate, the incubation rate for both the vector and the parasite and the probability of the vector feeding off a human host. These parameters are directly influenced by meteorological variables such as rainfall, temperature and humidity that give rise to differences in stability of disease transmission and seasonal variations in disease incidence.

Behavioral traits, genetic variation and immune status in the human population will also influence the degree of exposure and the disease outcome (WHO, 2014).

Around the world, the malaria situation is serious and getting worse. Malaria threatens the lives of $40 \%$ of the world's population - over 2200 million people. Each year, there are an estimated 300-500 million clinical cases. Malaria is estimated to kill more than 1 million people annually, the majority of whom are young children. Ninety per cent of malaria cases in the world occur in Africa south of the Sahara. Children under 5 years of age and pregnant women are the worst affected by malaria. It is one of the leading causes of death among young children.

Together with pneumonia, diarrhea, measles and malnutrition, malaria is responsible for over $70 \%$ of deaths in young children especially in developing countries. Malaria during pregnancy causes severe maternal illness and anemia, and is also associated with low birth weight among new born infants, a leading risk factor for infant mortality (UNICEF, 2000)

Malaria is thought to kill 1-1.5 million death worlds wide each year. Malaria is one of the major five courage of mankind in the developing countries, military conflicts and civil unrest, unfavorable ecological changes have greatly contributed to malaria epidemic un protected non immune and physically weakness refuge move in to malarias area.

Though to large extent, it has been eradicated from a large parts of North American and Europe, it is common in the most areas of the tropics. Distribution varies greatly from country to country and with the country themselves. In year( 1989), WHO declared malaria control to be a global priority due to worsening situation, and with 1993 the words assembly of with urged to increase control efforts, as well as control measures such as spraying with DDT coating mar shed with paraffin, drainage stagnant water and with wide spread of nets. There were an estimated 247 million episodes of malaria in 2006, with wide uncertainty interval from 189million to327 million cases86\%(112 million) cases were in Africa Region $80 \%$ of these cases were in 13 countries , and over half were in Nigeria, Democratic Republic of the Congo ,Ethiopia, United Republic of Tanzania ,Kenya and Sudan, Park, 2009) 


\section{JUSTIFICATION AND PROBLEM STATEMENT}

Malaria is a major public health problem, about half of the world's population lives under exposure. The problems are increasing day by day in magnitude and complexity because it is associated with low socio-economic status, which makes African women and Children particularly vulnerable.

In Sudan it is significant public health problem, since its leading cause of death, searching on methods of control and prevention of malaria is very important for health community.

This paper aim to verify methods of control and prevention as well as introducing solutions for the major problems of malaria in shendi Town.

\section{MATERIALS AND METHODS}

This descriptive cross sectional study was done in Shendi city during a year 2015 to assess methods of control and prevention of malaria in the city. Simple sample size of 192 was selected randomly out of 9000 households. Interviews questionnaires were conducted to collect data from household. Then the collected data was analyzed manually and computerize using both Microsoft excel and spss, then the results presented in tables and figures.

\section{RESULTS}

Table 1: Educational level of mothers

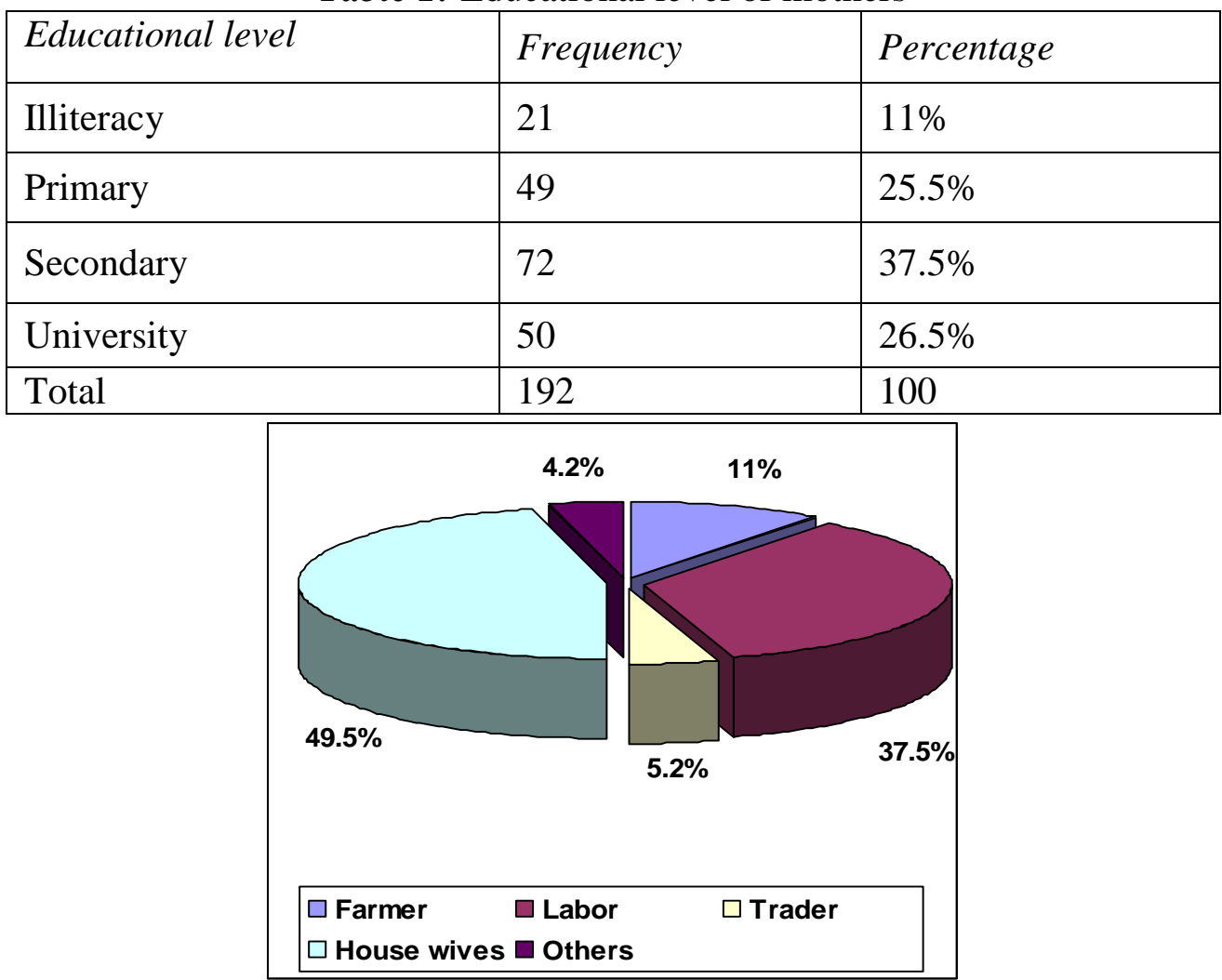

Figure 1: Occupational level of mothers in shendi 


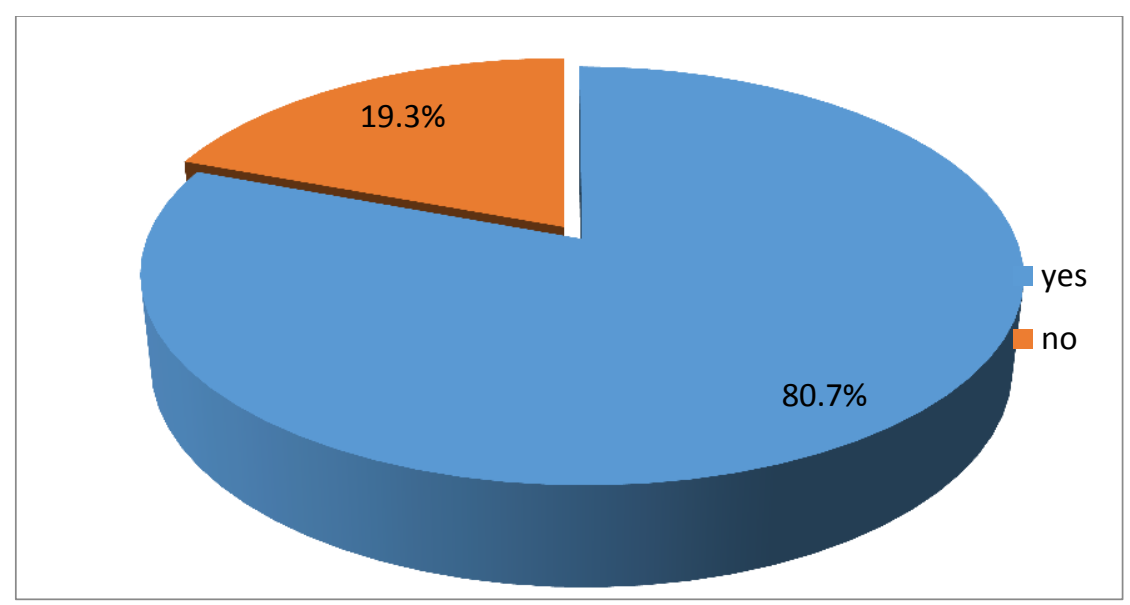

Figure 2: Presence of mosquito in the houses

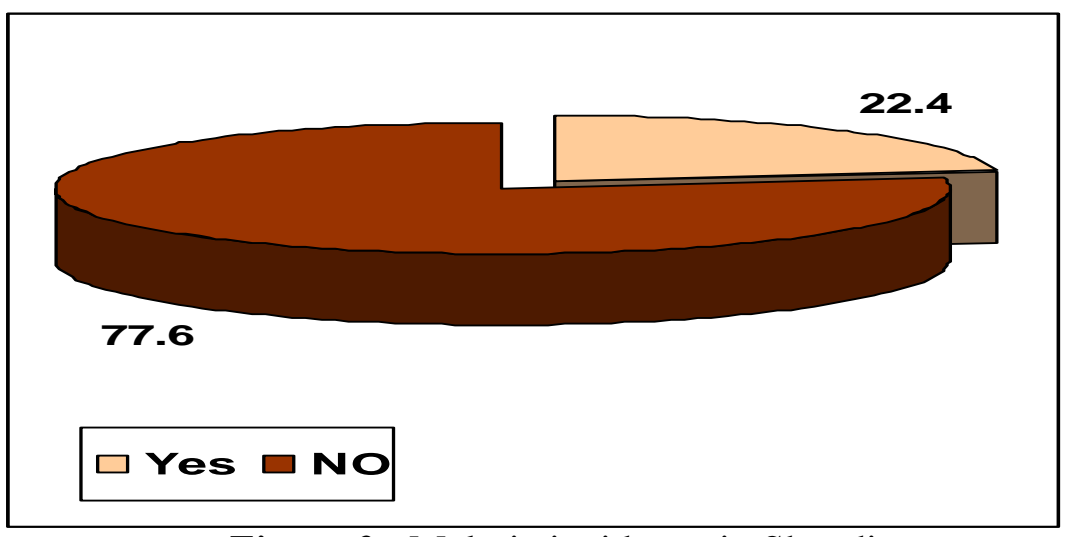

Figure 3: Malaria incidence in Shendi

Table 2: Mosquito breedingcites

\begin{tabular}{|l|l|l|}
\hline Cites of mosquito breeding & Frequency & Percentage \\
\hline Garden & 29 & $15.1 \%$ \\
\hline Tank & 83 & $43.3 \%$ \\
\hline toilets & 75 & $39.1 \%$ \\
\hline Others & 5 & $2.5 \%$ \\
\hline Total & 192 & $100 \%$ \\
\hline
\end{tabular}

Table 3: Personal protection against mosquito bites

\begin{tabular}{|l|l|l|}
\hline Personal protection & Frequency & Percentage \\
\hline Yes & 145 & $75.5 \%$ \\
\hline NO & 47 & $24.5 \%$ \\
\hline Total & 192 & 100 \\
\hline
\end{tabular}


Table 4: Mode of personal protection against mosquito bite

\begin{tabular}{|l|l|l|}
\hline Personal protection & Frequency & Percentage \\
\hline Impregnated mosquito net & 98 & $67.6 \%$ \\
\hline Repellents & 31 & $21 \%$ \\
\hline Screening & 4 & $2.8 \%$ \\
\hline Smoking & 11 & $7.6 \%$ \\
\hline Others & 1 & $1 \%$ \\
\hline Total & 145 & 100 \\
\hline
\end{tabular}

Table 5: Residual insecticide spraying during this year2015

\begin{tabular}{|l|l|l|}
\hline Residual insecticide & Frequency & Percentage \\
\hline Yes & 91 & $47.4 \%$ \\
\hline NO & 101 & $52.6 \%$ \\
\hline Total & 192 & 100 \\
\hline
\end{tabular}

\section{DISCUSSION}

The study showed that the main educational level of mothers was secondary level and university level with about $37.5 \%$ and $26 \%$ respectively these higher educational levels participate positively increase health awareness for the mother, utilization of health service and source reduction of mosquito breeding. Table (1) most occupational level of mother are house wives this may positively increase mothers participation in malaria control, due to their highly educational level and having enough knowledge about malaria, and also participates in mother's monitoring for their Children's health and nutritional status for them, In comparison with other study that was made in Sudan migrants families living in Saudi Arabia (Riyadh) in 1993 and was conducted by the international union for the scientific-study of population (IUSSP), this study became differ, while this study revealed that $70 \%$ of women were labors such as teachers and secretaries and $20 \%$ are house wives, while the above study showed that $49.5 \%$ were house wives, and $37.5 \%$ are labor. These factors and others increased skills and knowledge of prevention and control of malaria thus participate effectively in reduction of incidence of malaria in shendi town, Figure (1).

Most houses of the study area presented flying mosquitos this illustrated the need of strong program to control and prevention of malaria and need of protection against mosquitos bites, figure (2), and tanks represented the most appropriated location of breeding cites as well as toilets ,that means there were Anopheles as well as culex mosquitos ,thus the measures of prevention and control must include both of them , table(2)

The study reveals that most of population of shendi town uses personal protection against mosquito bite while significant number of them don't protect them self's against mosquito bites, 
this increase incidence of malaria because most of population suffer of malaria, table (3). This application of protection measures is the one of the factor that interrupts the transmission of malaria in the communities, thus decreasing the incidence of malaria in shendi city, especially the susceptible groups such as Children and pregnant women. In comparison of this study with other study that was done in 2005 by the American society and tropical medicine and hygiene for the personal protection against mosquitoes bite in this studCote d'Ivoire became nearly the same results, where this study revealed $80 \%$ of personal protection of mosquito bite in the community, while the above study showed $75.5 \%$ of personal protection from mosquito bites in shendi, table (3). Also the study reveals that the most dominant method of personal protection was impregnated mosquito net, while the repellents and used of smoke take place in such population, table(4).

Finally the study showed that the most of population said that: there was no larvacide spraying done by local health authorities to prevent breeding of mosquitos, table (5)

\section{CONCLUSIONS}

- The dominant educational level of study population was university and secondary school level.

- Most houses present flying mosquitos.

- Tanks represented the appropriate breading cite of mosquitos in city.

- Most of populations used personal protection against mosquito bite.

- Most of population said that there was no larvacide praying against mosquitos by local health authorities of shendi city

\section{RECOMMENDATIONS}

- Health authorities must improve the health education in the communities

- Ministry of health must provide the population with equipment and supplies such as bednet, drugs of malaria, repellents.

- Public health administration must eliminate the favorable places of mosquito breeding.

- Local public health authorities must adopt spraying program to control and prevent malaria.

\section{REFERENCES}

[1] Gordon c cook, Manson's (2004Tropical disease.

[2] Amerasinghe, F. (2006). Water, health and environment. In Giordano, London, UK.

[3] Gallup, J.L., and Sachs, J.D. (2001). The economic burden of malaria. American Journal of Tropical Medicine and Hygiene 64: 85-96

[4] WHO. World Health Organization. (2007). Fact Sheet No. 97, May 2007. http://www.who.int/mediacentre/factshee ts/fs094/en/index.html (Date Accessed, July 30, 2007).

[5] WHO (2014) Malaria Control Manual. A manual for Elimination Scenario planning, Imperial College. London Global Malaria program me .World Health Organization

[6] W H O (2004) report of Malaria and travel. 
[7] United Nations Children!s Fund (UNICEF) with cooperation of the World health organization (2000) Promoting Rational use of Drugs. New York

[8] WHO. World Health Organization. (2000). World Health Organization

[9] Expert Committee on Malaria, Twentieth Report, (WHO Technical Report Series, No. 892).Geneva.

[10] K. Park John Everett (2009): "Park's Text Book of Preventive \& Social Medicine "20th ed. India.

[11] The National protocol for treatment of malaria (2010) Khartoum. 